Dr. Eike Alexander v. Boetticher und Dr. Daniel Heimes"

\title{
Die Anpassung des rheinland-pfälzischen Landesarchivgesetzes an die DSGVO
}

\section{Einleitung}

Seit 25. Mai 2018 gilt die Verordnung (EU) 2016/679 des Europäischen Parlaments und des Rates vom 27. April 2016 zum Schutz natürlicher Personen bei der Verarbeitung personenbezogener Daten, zum freien Datenverkehr und zur Aufhebung der Richtlinie 95/46/EG (EU-Datenschutzgrundverordnung - DSGVO) als unmittelbares Recht gemäß Art. 288 Abs. 2 AEUV in der gesamten Europäischen Union, soweit ihr Anwendungsbereich betroffen ist und damit auch für Archive. ${ }^{1}$

Mit dem Zweiten Landesgesetz zur Änderung des Landesarchivgesetzes vom 11. Februar $2020^{2}$ wurde das rheinland-pfälzische Landesarchivgesetz (LArchG RhPf) an die DSGVO angepasst, nachdem dieses vom Landtag in der Sitzung vom 29. Januar 2020 einstimmig angenommen worden war. ${ }^{3}$ Dabei wurde insbesondere von der Derogations-Möglichkeit Gebrauch gemacht, die Art. 89 Abs. 3 DSGVO für im „öffentlichen Interesse“ liegende Archivzwecke vorsieht. Vorbild für die Anpassung des Landesarchivgesetzes war ein Entwurf des Ausschusses Archive und Recht der Konferenz der Leiterinnen und Leiter der Archivverwaltungen des Bundes und der Länder (KLA). Daraus fertigten die Autoren in ihrer Funktion als Referenten der Landesarchivverwaltung Rheinland-Pfalz die inhaltlich maßgebliche und im Umfang ausführlichere Vorlage für den Referentenentwurf des Ministeriums für Wissenschaft, Weiterbildung und Kultur Rheinland-Pfalz. Dabei konnte vor allem an bereits bestehende Regelungen zu den Betroffenenrechten ( $\$ 4$ LArchG Rh-Pf) angeknüpft werden, die sich bereits seit längerer Zeit bewährt hatten. ${ }^{4}$ Hinzuweisen ist ferner auf den neu aufgenommenen $\$ 6$ Landesdatenschutzgesetz, der als Pendant zu $\$ 7$ Abs. 1. S. 1

* Die Verfasser gehören in der Landesarchivverwaltung Rheinland-Pfalz der Organisationeinheit "Stabstelle Übergreifende Fachaufgaben" an und teilen sich den Aufgabenbereich zu Rechtsfragen im Archivwesen. Zudem sind sie für den Datenschutz zuständig.

1 Steinhauer, Handbuch Europäisches und deutsches Datenschutzrecht, 2019, S. 699, 701.

2 Gemeinsames Amtsblatt des Ministeriums für Bildung und des Ministeriums für Wissenschaft, Weiterbildung und Kultur vom 23.03.2020, S. $78 \mathrm{f}$.

3 Landtag Rheinland-Pfalz - 17. Wahlperiode - 98. Sitzung, 29.01.2020, S. 6591. Die Beiträge der eher knappen Aussprache im Landtag finden sich ebd., S. 6588-6591.

$4 \mathrm{Zu}$ den (bisherigen) Betroffenenrechten: Hausmann, Archivrecht. Ein Leitfaden, 2016, S. 93 96; Manegold, Archivrecht. Die Archivierungspflicht öffentlicher Stellen und das Archivzu- 
LArchG Rh-Pf klargestellt, dass eine Löschung personenbezogener Daten erst zulässig ist, nachdem die Unterlagen dem zuständigen Archiv angeboten worden sind. ${ }^{5}$

Ausgehend von einem Überblick über die wichtigsten Artikel der DSGVO für Archive sollen im Folgenden die Änderungen des Landesarchivgesetzes vorgestellt werden.

II. Zentrale Begriffe der DSGVO für das Archivwesen

\section{Personenbezogene Daten}

Der Ausdruck „personenbezogene Daten“ i.S.v. Art. 4 Nr. 1 DSGVO bezeichnet „alle Informationen, die sich auf eine identifizierte oder identifizierbare natürliche Person (im Folgenden ,betroffene Person') beziehen: als identifizierbar wird eine natürliche Person angesehen, die direkt oder indirekt, insbesondere mittels Zuordnung zu einer Kennung wie einem Namen, zu einer Kennnummer, zu Standorten, zu Standortdaten, zu einer Online-Kennung oder zu einem oder mehreren besonderen Merkmalen, die Ausdruck der physischen, psychologischen, genetischen, psychischen, wirtschaftlichen, kulturellen oder sozialen Identität dieser natürlichen Person sind, identifiziert werden kann." Damit ist klargestellt, dass nicht nur das von seinem Verständnis viel engere personenbezogene Archivgut in den Anwendungsbereich der DSGVO fällt, sondern - unter Umständen - auch weitere, am Rande erwähnte, sich in Archivgut findende personenbezogene Daten. ${ }^{6}$

\section{Archivierung als „Verarbeitung“}

Nach Art. 4 Nr. 2 DSGVO meint „Verarbeitung“ „jeden mit oder ohne Hilfe automatisierter Verfahren ausgeführten Vorgang oder jede solche Vorgangsreihe im Zusammenhang mit personenbezogenen Daten wie das Erheben, das Erfassen, die Organisation, das Ordnen, die Speicherung, die Anpassung oder Veränderung, das Auslesen, das Abfragen, die Verwendung, die Offenlegung durch Übermittlung, Verbreitung oder eine andere Form der Bereitstellung, den Abgleich oder die Verknüpfung, die

gangsrecht des historischen Forschers im Lichte der Forschungsfreiheitsverbürgung des Art. 5 Abs. 3 GG, 2002, S. 319-325.

In der DSGVO ist statt „Betroffenen“ von „betroffenen Personen“ die Rede. Bei der Anpassung des LArchG Rh-Pf ist eine sprachliche Anpassung nicht erfolgt, wird aber bei der nächsten Novellierung nachgeholt. In diesem Aufsatz wird hingegen schon „betroffenen Personen“ die Rede sein.

5 Kugelmann-Richter, Landesdatenschutzgesetz Rheinland-Pfalz, 2020, $\$ 6$ Rn. 1-7.

6 Zur Unterscheidung personenbezogenes Archivgut und personenbezogene Daten, vgl:. Hausmann, Landesarchivgesetz, S. 12, 16; Manegold, Archivrecht. Die Archivierungspflicht öffentlicher Stellen und das Archivzugangsrecht des historischen Forschers im Lichte der Forschungsfreiheitsverbürgung des Art. 5 Abs. 3 GG, 2002, S. 278; kritisch zu dieser Unterscheidung bei der online-Archivierung: Diesterhöft, Digitale Bibliotheken, 2014, S. 51, 73 f.; vgl. dazu insgesamt auch unter 2. Archivierung als „Verarbeitung“. 
Einschränkung oder die Vernichtung “ von personenbezogenen Daten. Diese Aufzählung ist nicht abschließend, umfasst aber bereits einen sehr weiten sachlichen Anwendungsbereich. ${ }^{7}$ In einem Archiv unterfallen unter anderem folgende Arbeitsschritte mit personenbezogenen Daten dem Verarbeitungsbegriff: die Anbietung und Übernahme, das Ordnen, die Speicherung, das Erfassen bzw. die Erschließung, die Anpassung oder Veränderung, das Auslesen, die Verwendung, die Nutzung und Bereitstellung in jeglicher Form sowie das Löschen oder die Vernichtung.

Wichtig ist, dass nach Art. 2 Abs. 1 DSGVO die Verordnung „für die ganz oder teilweise automatisierte Verarbeitung personenbezogener Daten sowie für die nichtautomatisierte Verarbeitung personenbezogener Daten“ gilt, „die in einem Dateisystem gespeichert sind oder gespeichert werden sollen." Die DSGVO umfasst also nicht nur personenbezogene Daten, wenn diese (nur) digital vorliegen. Maßgeblich ist vielmehr, „dass entweder ein mit Hilfe automatisierter Verfahren ausgeführter Vorgang bzw. eine Vorgangsreihe im Zusammenhang mit personenbezogenen Daten vorliegt oder die Daten Teil einer nach personenbezogenen Kriterien strukturierten Sammlung sind, sodass

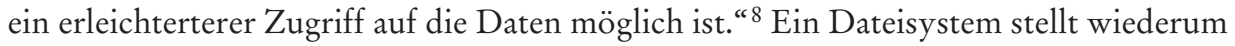
„jede strukturierte Sammlung personenbezogener Daten“ dar, die nach bestimmten Kriterien zugänglich sind, unabhängig davon, ob diese Sammlung zentral, dezentral oder nach funktionalen oder geografischen Gesichtspunkten geführt wird“, Art. 4 Nr. 6 DSGVO. Nach Ansicht des Europäischen Gerichtshofs muss „eine solche Sammlung nicht aus spezifischen Kartotheken oder Verzeichnissen oder anderen der

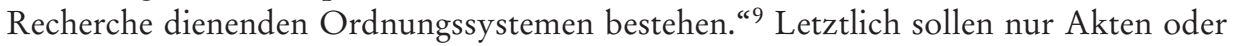
Aktensammlungen (samt Deckblätter), die nicht nach bestimmten Kriterien geordnet sind, nicht in den Anwendungsbereich der DSGVO fallen, ErwGr 15 S. 3. Einen solchen Zustand sollte es dem Anspruch nach in einem Archiv allerdings nicht geben.

\section{Privilegierung der Archivzwecke}

1. Privilegierungen der „in öffentlichem Interesse liegenden Archivzwecke“

Die DSGVO gilt nicht für die personenbezogenen Daten Verstorbener, sodass große Teile der Archivbestände nicht in ihren Geltungsbereich fallen. ${ }^{10}$ Darüber hinaus hat

7 Sydow-Reimer, Europäische Datenschutzgrundverordnung, 2. Aufl. 2018, Art. 4 Rn. 43; zu den einzelnen Verarbeitungsbegriffen: Sydow-Reimer, Europäische Datenschutzgrundverordnung, 2. Aufl. 2018, Art. 4 Rn. 55-76.

8 Sydow-Reimer, Europäische Datenschutzgrundverordnung, 2. Aufl. 2018, Art. 2 Rn.1; EuGH, Urt. v. 10.07.2018 - C-25/17, ECLI:EU:C:2018:551 = EuZW 2018, 897 Tz. 53 - Jehovan todistajat/Datenschutzkommission.

9 EuGH, Urt. v. 10.07.2018 - C-25/17, ECLI:EU:C:2018:551 = EuZW 2018, 897 Tz. 62 - Jehovan todistajat/Datenschutzkommission.

10 ErwGr 27 S.1. und ErwGr 158 S. 1 DSGVO; Plath-Grages, DSGVO/BDSG, 3. Auflage 2018, Art. 89 Rn. 10. Dass Daten von Verstorbenen für Archive aber dennoch von datenschutzrechtlichem Belang sein können, zeigt die aktuelle Diskussion um die online-Stellung von Personenstandsregistern, vgl.: Engelbrecht, DÖV 2017, 393; Nestl, RuZ 2020, 5 , 7 ff. 
die DSGVO an einigen Stellen Privilegierungen der „in öffentlichem Interesse liegenden Archivzwecke" vorgenommen, sodass in diesen Fällen die Bestimmungen der DSGVO auf die Datenverarbeitung nicht anzuwenden sind und keine Anpassung im LArchG Rh-Pf notwendig war: Art. 5 Abs. 1 lit. b und lit. e DSGVO entheben Archive von den in der DSGVO grundsätzlich vorgegebenen Grundsätzen der Zweckbindung und Speicherbegrenzung, ${ }^{11}$ denn die Weiterverarbeitung personenbezogener Daten für im öffentlichen Interesse liegende Archivzwecke wird prinzipiell als vereinbar mit den ursprünglichen Erhebungszwecken und damit als rechtmäßig angesehen. ${ }^{12}$ Art. 14 Abs. 5 lit. b DSGVO sieht eine Ausnahme für Archive bei den umfangreichen Informationspflichten vor, wenn die personenbezogenen Daten nicht bei der betroffenen Person erhoben wurden. ${ }^{13}$ Besonders wichtig erscheint die Ausnahme in Art. 17 Abs. 3 lit. d DSGVO, der entgegen der - vor allem in den ersten Monaten seit Inkrafttreten der DSGVO häufig anzutreffenden Vorstellung bei einigen Behörden und Bürgern eine Löschung personenbezogener Unterlagen nach Art.17 Abs. 1 DSGVO ${ }^{14}$ ausschließt, wenn deren Verarbeitung für Archivzwecke „im öffentlichen Interesse erforderlich ist. " 15

Was die öffentlichen Interessen genau sein sollen, wird hingegen in der DSGVO nicht definiert. ${ }^{16}$ Den einzigen Anhaltspunkt liefert ErwGr 158, der beispielhaft einige archivische Tätigkeiten, wie die Archivierung zur Aufarbeitung des Unrechts totalitärer Regime und Völkermord, aufzählt. ${ }^{17}$ Man wird aber mit Recht annehmen dürfen, dass Archive von staatlichen und von anderen öffentlich-rechtlich verfassten Rechtspersonen (z.B. Kommunen) grundsätzlich im öffentlichen Interesse tätig sind. ${ }^{18}$

Hingewiesen sei an dieser Stelle auf den Vortrag des Würzburger Stadtarchivars Axel Metz auf dem letztjährigen Archivtag in Suhl zu diesem Thema, der demnächst verschriftlicht werden soll.

11 Nach dem Grundsatz der Zweckbindung darf die Verarbeitung personenbezogener Daten nur zu zuvor eindeutig festgelegten, legitimen Zwecken erfolgen; der eng damit zusammenhängende Grundsatz der Speicherbegrenzung gibt vor, dass eine Speicherung nur so lange erfolgen soll, wie es für die Durchführung der vorab bestimmten Zwecke unverzichtbar ist, ErwGr 39 S. 6 f. DSGVO; Schantz/Wolff, Das neue Datenschutzrecht, 2017, Rn. 444.

12 ErwGr 50 S. 4 DSGVO; Schantz/Wolff, Das neue Datenschutzrecht, 2017, Rn. 413 f.

13 Für Daten, die Archive von ihren Nutzern selbst erfassen, gilt allerdings Art. 14 DSGVO weiterhin. Zum Umgang mit diesen Daten, vgl.: Steinhauer, Handbuch Europäisches und deutsches Datenschutzrecht 2019, S. 699, 710 f.

14 Das Recht auf Löschung beinhaltet das vom EuGH entwickelte „Recht auf Vergessenwerden“, EuGH, Urt. v. 13.05.2014 - C-131/12, ECLI:EU:C:2014:317 = GRUR 2014, 895 Tz. 89 ff. - Google Spain, ist aber mit diesem nicht identisch (ErwGr 66).

15 Sydow-Penker, Europäische Datenschutzgrundverordnung, 2. Aufl. 2018, Art. 17 Rn. 66.

16 Ehmann/Selmayr-Raum, Datenschutzgrundverordnung, 2. Aufl. 2018, Art. 89 Rn. 23.

17 ErwGr 158 S. 4 DSGVO.

18 Sydow-Hense, Europäische Datenschutzgrundverordnung, 2. Aufl. 2018, Art. 89 Rn. 17; Birk, Sächsisches Archivblatt 2 2018, 11, 12. Höchst umstritten ist allerdings, ob sich auch Privatarchive wie Unternehmensarchive auf die Privilegierung des öffentlichen Interesses stützen können, vgl.: Schantz/Wolff, Das neue Datenschutzrecht, 2017, S. 409; Plath-Grages, DSGVO/BDSG, 3. Auflage 2018, Art. 89 Rn. 11. 
2. Bedingte Privilegierungen der „in öffentlichem Interesse liegenden Archivzwecke“

Von besonderer Bedeutung für die Arbeit der Archive ist Art. 9 DSGVO, der die Verarbeitung besonderer Kategorien personenbezogener Daten regelt. Dabei handelt es sich um personenbezogene Daten, „aus denen die rassische und ethnische Herkunft, politische Meinungen, religiöse oder weltanschauliche Überzeugungen, die Gewerkschaftszugehörigkeit hervorgehen" sowie genetische Daten, biometrische Daten zur eindeutigen Identifizierung einer natürlichen Person, Gesundheitsdaten oder Daten zum Sexualleben oder der sexuellen Orientierung, Art. 9 Abs. 1 DSGVO. Art. 9 Abs. 1 DSGVO untersagt ausdrücklich die Verarbeitung dieser besonders sensiblen Daten, während Art. 9 Abs. 2 lit.j DSGVO Voraussetzungen für eine Ausnahme von im öffentlichen Interesse liegenden Archivzwecken nennt.

Zunächst bedarf es einer Ermächtigung auf „Grundlage des Unionsrechts oder des Rechts des Mitgliedstaates“, wozu auch das Landesrecht und damit das Landesarchivgesetz zählt. ${ }^{19} \$ 7$ Abs. 2 LArchG Rh-Pf wurde dahingehend ergänzt, dass auch Unterlagen anzubieten sind, die besondere Kategorien personenbezogener Daten enthalten. Der neu geschaffene $\ 8$ a LArchG Rh-Pf ermöglicht nun die Verarbeitung besonderer Kategorien personenbezogener Daten. ${ }^{20}$ Ohne eine solche ausdrückliche Ermächtigung im LArchG Rh-Pf wäre dies nicht gestattet. ${ }^{21}$

Gleichzeitig ist nach den Vorgaben von Art. 9 Abs. 2 lit. j DSGVO aber auch dafür Sorge zu tragen, dass die entsprechende Regelung ,in angemessenem Verhältnis zu dem verfolgten Ziel steht, den Wesensgehalt des Rechts auf Datenschutz wahrt und angemessene und spezifische Maßnahmen zur Wahrung der Grundrechte und Interessen der betroffenen Person vorsieht." Dies wird u.a. durch die archivgesetzlichen Sperrfristen nach $₫ 3$ Abs. 3 LArchG Rh-Pf erreicht, die sogar über den Tod und damit die Bestimmungen der Datenschutz-Grundverordnung hinausgehen ${ }^{22}$, eine etwaige Versagung der Archivbenutzung infolge einer entsprechenden Rechtsgüterabwägung nach

19 LT-Drs. 17/10671, S. 10.

20 Durch die Aufnahme eines entsprechenden Verweises in $\$ 2$ Abs. 3 S. 2 LArchG Rh-Pf wird klargestellt, dass die Verarbeitung besonderer Kategorien personenbezogener Daten auch für die von den mit Zustimmung des für Archivwesen zuständigen Ministeriums unterhaltenen eigenen fachlich betreuten Archiven der in $\ 1$ Abs. 1 S. 1 und 2 LArchG Rh-Pf genannten juristischen Personen des öffentlichen und privaten Rechts zulässig ist, LT-Drs. 17/10671, S. 7.

21 So auch: Heilmann, Die Anpassung des Niedersächsischen Archivgesetzes an die Vorgaben der Datenschutz-Grundverordnung, in: Archiv-Nachrichten Niedersachsen 23, 2018, 139, $140 \mathrm{f}$.

22 Die Sperrfristen werden in $₫ 3$ Abs. 3 LArchG Rh-Pf geregelt: Nach $₫ 3$ Abs. 1 S. 1 darf Archivgut erst 30 Jahre nach Entstehung der Unterlagen benutzt werden. „Soweit es sich auf natürliche Personen bezieht, darf es erst zehn Jahre nach deren Tod, oder, wenn das Todesjahr dem Archiv nicht bekannt ist, erst 100 Jahre nach der Geburt des Betroffenen benutzt werden; wenn weder das Jahr der Geburt noch das Jahr des Todes des Betroffenen bekannt ist, gilt eine Frist von 60 Jahren nach Entstehung der Unterlagen“, 33 Abs. 1 S. 2 LArchG. Unterlagen, die aufgrund von Rechtsvorschriften geheim zu halten sind, sind erst 60 Jahre nach ihrer Entstehung benutzbar, $\mathbb{\$} 3$ Abs. 1 S. 4 LArchG. Die Möglichkeiten zur Sperrfristverkürzung sind in $\$ 3$ Abs. 4 LArchG geregelt. $\mathrm{Zu}$ archivrechtlichen Sperrfristen allgemein: 
\3 Abs. 2 Nr. 2 LArchG Rh-Pf, wonach auch nach Ablauf der Sperrfristen eine Verpflichtung zur Einschränkung und Versagung der Benutzung besteht, wenn Grund zur Annahme besteht, dass schutzwürdige Belange betroffener Personen oder Dritter entgegenstehen, sowie speziell das Verarbeitungsverzeichnis des neu einzufügenden $\ 8 \mathrm{a}$ LArchG Rh-Pf. ${ }^{23}$

$\mathrm{Zu}$ den geforderten „angemessenen und spezifischen Maßnahmen“ können, je nach den Umständen des Einzelfalls, gehören:

- Technische und organisatorische Maßnahmen, um sicherzustellen, dass die Verarbeitung besonderer Kategorien personenbezogener Daten datenschutzkonform erfolgt, sowie Maßnahmen, die gewährleisten, dass nachträglich überprüft und festgestellt werden kann, ob und von wem personenbezogene Daten eingegeben, verändert oder entfernt sind. Die Verarbeitung solcher Daten erfolgt ausschließlich durch beamtete Personen oder solche, die nach dem Verpflichtungsgesetz vom 2. März 1975 verpflichtet sind, was jeweils auch über das Dienst- bzw. Beschäftigungsverhältnis hinaus einen Schutz darstellt.

- Die Sensibilisierung der an den Verarbeitungsvorgängen Beteiligten, was durch regelmäßige Schulungen und Hinweise erfolgt.

- Die Beschränkung des Zugangs zu den personenbezogenen Daten innerhalb der verantwortlichen Stelle und von Auftragsverarbeitern. Zugang zum Magazin erhalten nur Personen, bei denen dies für ihre Tätigkeit erforderlich ist. Zudem ist es möglich, festzustellen mit welchem einem Mitarbeiter zuordenbaren Transponder eine Magazintür wann geöffnet wurde. Hinsichtlich der über diese Unterlagen gespeicherten Metadaten ist anhand des personalisierten und in seinen Lese- und Schreibrechten gestuften Zugangs eine Kontrolle hinsichtlich Eingabe, Veränderung und Entfernung (Löschung) möglich.

- Die (gebührenpflichtige) Anonymisierung und Verschlüsselung personenbezogener Daten vor einer Nutzung von besonders sensiblen personenbezogenem Archivgut. Reproduktionen von entsprechendem Archivgut werden generell nicht unanomyisiert versendet.

- Die dauerhafte Sicherstellung der Vertraulichkeit, Integrität, Verfügbarkeit und Belastbarkeit der Systeme, Dienste im Zusammenhang mit der Verarbeitung personenbezogener Daten einschließlich der Fähigkeit, die Verfügbarkeit personenbezogener Daten und den Zugang zu ihnen bei einem physischen oder technischen Zwischenfall rasch wiederherzustellen. Die Landesarchivverwaltung Rheinland-Pfalz verfügt hinsichtlich der digitalen Daten über ein Backup-System zur Sicherung der Server. Hinsichtlich der analogen Unterlagen nimmt sie für ausgewählte Unterlagen zwar an der Sicherungsverfilmung des Bundes und der Einlagerung auf Filmen im

Hausmann, Archivrecht. Ein Leitfaden, 2016, S. 80-92; Brüdegam, Schutzfristen - Festlegung und Verkürzung, 2012, S. 61.

23 LT-Drs. 17/10671, S. 10. Unterlagen, die die Intimsphäre berühren, werden in Rücksprache mit dem Beauftragten für Datenschutz und Informationsfreiheit auf Grundlage der „Dreisphärentheorie“ des Bundesverfassungsgerichts generell nicht vorgelegt, Telefonat vom 29.11.2019. 
Barbarastollen (der Zentrale Bergungsort der Bundesrepublik Deutschland in der Nähe von Freiburg im Breisgau) teil, jedoch liegt der Schwerpunkt auf den Unterlagen des Alten Reichs. Modernes Schriftgut wird nur in Auswahl verfilmt. Dies bedeutet, dass hinsichtlich der analogen Unterlagen mit Datenschutzrelevanz an dieser Stelle insbesondere Brandschutzvorkehrungen zu nennen sind.

- Zur Gewährleistung der Sicherheit der Verarbeitung die Einrichtung eines Verfahrens zur regelmäßigen Überprüfung, Bewertung und Evaluierung der Wirksamkeit der technischen und organisatorischen Maßnahmen oder spezifischen Verfahrensregelungen, die im Falle einer Übermittlung oder Verarbeitung für andere Zwecke die Einhaltung der datenschutzrechtlichen Vorgaben sicherstellen. ${ }^{24}$ Darüber hinaus ist vor der Nutzung von besonderen Kategorien personenbezogener Daten stets eine Verpflichtungserklärung zu unterzeichnen, die eine grundsätzliche Anonymisierung bei einer etwaigen Veröffentlichung und das Verbot der Selbstanfertigung von Reproduktionen vorsieht. ${ }^{25}$

Die ebenfalls in Art. 9 Abs. 2 lit. j DSGVO geforderten „für im öffentlichen Interesse liegende Archivzwecke" finden sich u.a. in der Sicherung berechtigter Belange der Bürger, z.B. bei der Entschädigung von Heimkindern, der Aufarbeitung von Verfolgungsschicksalen, insbesondere der NS-Zeit, der Medizinforschung und der retroperspektiven Aufarbeitung von gesellschaftlichen Diskriminierungsformen, was auch präventiv in die Gesellschaft zurückwirken soll. ${ }^{26}$ Diese Aufzählung ist allerdings keinesfalls abschließend zu verstehen.

\section{Derogation einzelner Artikel}

Art. 89 DSGVO fungiert zwar nicht als eigener Erlaubnistatbestand, ermöglicht aber in Abs. 3 die Derogation für die Verarbeitung im öffentlichen Interesse liegenden Archivzwecke der Art. 15, 16, 18, 19, 20 und 21, also die Ausnahmeregelung der Geltung dieser Artikel. Die Derogationsmöglichkeit besteht allerdings nur, wenn die Arbeit privilegierter Archive ansonsten massiv beeinträchtigt wäre und stellt keine Generalvollmacht für die Verarbeitung personenbezogener Daten durch privilegierte Archive dar. Gleichwohl sollen „überschießende Effekte im Einzelfall“ hinzunehmen sein. ${ }^{27}$

Durch das Landesarchivgesetz waren diese Artikel daher ausdrücklich außer Kraft zu setzen, wobei an bestehende Regelungen zu Betroffenenrechten in $\$ 4$ LArchG Rh-

24 LT-Drs. 17/10671, S. 10 f.

25 Die Verpflichtungserklärung ist $₫ 22$ Abs. 5 LDSG nachgebildet, wonach personenbezogene Daten nur veröffentlicht werden dürfen, wenn die betroffene Person eingewilligt hat oder dies für die Darstellung von Forschungsergebnissen über Ereignisse der Zeitgeschichte unerlässlich ist und überwiegende schutzwürdige Interessen der betroffenen Person nicht entgegenstehen, vgl. hierzu: Kugelmann-Smolle, Landesdatenschutzgesetz, $\$ 22$ Rn. $62 \mathrm{ff}$.

26 Vgl. LT-Drs. 17/10671, S. 11.

27 Plath-Grages, DSGVO/BDSG, 3. Auflage 2018, Art. 89 Rn. 6; Schantz/Wolff, Das neue Danteschutzrecht, 2017, S. 136 f.; Paal/Pauly-Pauly, DSGVO BDSG, 2. Aufl. 2018, Art. 89 Rn. 1. 
Pf angeknüpft werden konnte. Die Inanspruchnahme dieser Privilegierungen werden nach Art. 89 Abs. 1 DSGVO allerdings von geeigneten Garantien für die Rechte und Freiheiten betroffener Personen abhängig gemacht, mit denen sichergestellt wird, dass technische und organisatorische Maßnahmen bestehen, durch die der Grundsatz der Datenminimierung (Art. 5 Abs. 1 lit. c DSGVO) gewährleistet wird. ${ }^{28}$ Dieser Grundsatz besagt, dass Daten nicht unnötig im Sinne einer Vorratsdatenspeicherung angesammelt werden sollen, und Archivierung auf das tatsächlich Notwendige zu beschränken ist. ${ }^{29}$ Bereits vor Einführung der DSGVO war aber schon anerkannt, dass „Archive nur die nach archivfachlichen Kriterien als archivwürdig bestimmen Unterlagen übernehmen, so dass unabhängig von der Sensibilität der Daten nur ein kleiner Teil der Datenbestände archiviert wird. “30 An dieser Position hält die Landesarchivverwaltung - gegen kritische Stimmen - auch im digitalen Zeitalter fest und will keinesfalls ganze Datenpools übernehmen und eine neue „Jäger- und Sammlermentalität“ begründen. ${ }^{31}$

Darüber hinaus sind gerade in den letzten Jahren Archivierungsmodelle und Dokumentationsprofile (z.B. für Personalakten, Patientenakten oder Steuerakten der Finanzämter) entstanden, um dadurch eine aussagekräftigere Überlieferung zu bilden. ${ }^{32}$ Die Übernahmequote liegt dabei, sofern keine pauschale Vernichtungsgenehmigung nach $\$ 7$ Abs. 4 LArchG Rh-Pf erteilt wird, welche die Quote auf null reduziert, grundsätzlich im Bereich von unter 0,1 Prozent bis ca. 10 Prozent. Dabei sind Ausnahmen nach oben bei Anbietungen möglich, z.B. wenn der Gegenstand ein besonders hohes und anhaltendes mediales Interesse erzeugt hat. Bespielhaft soll hier die Staatsanwaltschaft Koblenz als anbietungspflichtige Stelle mit besonders vielen und sensiblen personenbezogenen Daten genannt werden. Diese leitet zurzeit etwas mehr als 80.000 Ermittlungsverfahren pro Jahr ein. Das Archivierungsmodell, das hier nicht im Einzelnen dargestellt werden soll, steht für eine Übernahmequote von unter 0,1 Prozent.

Ferner verpflichtet $\int 9$ Abs. 2 LArchG Rh-Pf die Archive, das Archivgut durch organisatorische, technische und personelle Maßnahmen vor unbefugter Benutzung, Beschädigung und Verlust zu schützen.

28 Birk, Sächsisches Archivblatt 2 2018, 11, 12; Paal/Pauly-Pauly, DSGVO BDSG, 2. Aufl. 2018, Art. 89 Rn. 2 und Rn. 11.

29 Plath-Grages, DSGVO/BDSG, 3. Aufl. 2018, Art. 89 Rn.2; Paal/Pauly-Pauly, DSGVO BDSG, 2. Aufl. 2018, Art. 89 Rn. 12.

30 Hausmann, Landesarchivgesetz, S. 23.

31 Pawelletz, Internationales Archivsymposium in Maastricht (2014). Bewertung und Überlieferungsbildung, 2015, S. 23, $27 \mathrm{f}$.

32 Pawelletz, Internationales Archivsymposium in Maastricht (2014). Bewertung und Überlieferungsbildung, 2015, S. 23, 24 f.; Becker, Archivrecht für die Praxis 2017, S. 58, 70. Zum Archivierungsmodell für Personalakten, vgl.: Richtlinien zur Übernahme von Personalakten der staatlichen Behörden des Landes Rheinland-Pfalz durch die Landesarchivverwaltung Rheinland-Pfalz, abrufbar unter: https://www.landeshauptarchiv.de/unser-auftrag/staatliche -behoerden/anbietung-und-aussonderung/richtlinien-fuer-die-anbietung-von-personalakte n, zuletzt abgerufen am 19.06.2020. 


\section{a) Art. 15 DSGVO}

Art. 15 DSGVO regelt das Auskunftsrecht der betroffenen Personen. Die Bestimmung räumt jeder Person das uneingeschränkte Recht ein, von dem Verantwortlichen, also auch von einem öffentlichen Archiv, eine Bestätigung darüber zu verlangen, ob von dieser personenbezogene Daten verarbeitet werden. Wie bereits erwähnt, stellt so gut wie jede Form der Archivierung eine „Verarbeitung von Daten“ dar.

Allerdings werden Daten in Archiven zu einem völlig anderen Zweck verarbeitet als in anderen Stellen. Aufgabe eines Archivs ist es nämlich nicht (nur), Daten zu erfassen, die es zur Wahrnehmung seiner Aufgaben benötigt. Stattdessen werden in einem Archiv Daten verarbeitet, die andere Behörden zur Wahrnehmung ihrer Aufgaben nicht mehr benötigen. Die Verarbeitung in einem Archiv erfolgt vor allem, um diese Daten langfristig zu sichern und - unter Einhaltung strenger archivgesetzlich festgelegter Datenschutzvorschriften - betroffenen Personen und anderen Berechtigten, wie Forschern, zugänglich zu machen.

Das umfassende Auskunftsrecht nach Art. 15 DSGVO ginge weit über die Kernaufgabe der Archive hinaus und würde für diese angesichts der damit verbundenen Aufwände eine ernsthafte Beeinträchtigung darstellen. Zudem sah das LArchG bereits in seiner bisherigen Fassung ein umfassendes Auskunftsrecht betroffener Personen vor ( $\int 4$ Abs. 1 LArchG Rh-Pf), das sowohl den Bedürfnissen der betroffenen Person als auch den Aufgaben der Archive gerecht wird. ${ }^{33}$ Eine betroffene Person kann demnach - unabhängig von den in $\$ 3$ Abs. 3 LArchG Rh-Pf geregelten Sperrfristen - Auskunft oder Einsicht verlangen, soweit sich Archivgut auf ihn bezieht und \$3 Abs. 2 LArchG Rh-Pf nicht entgegensteht. ${ }^{34}$ Dabei hat der Antragsteller die Position als betroffene Person glaubhaft darzulegen, um eine missbräuchliche Archivbenutzung auszuschließen. Außerdem sind Angaben erforderlich, die das Auffinden des ihn betreffenden Archivguts ohne unangemessenen Aufwand ermöglicht. ${ }^{35}$ Daher wurde eine Ergänzung in $\int 4$ Abs. 1 S. 3 LArchG Rh-Pf aufgenommen, durch die die Geltung von Art. 15 DSGVO ausgeschlossen wurde.

33 LT-Drs. 17/1067, S. 7 f.

$34 \rrbracket 3$ Abs. 2 LArchG: „Die Benutzung ist einzuschränken oder zu versagen, soweit

1. Grund zur Annahme besteht, dass das Wohl der Bundesrepublik Deutschland oder eines ihrer Länder gefährdet würde, oder

2. Grund zur Annahme besteht, dass schutzwürdige Belange Betroffener oder Dritter entgegenstehen, oder

3. der Erhaltungszustand des Archivguts gefährdet würde, oder

4. die Geheimhaltungspflicht nach $\$ 203$ Abs. 1 bis 3 des Strafgesetzbuches oder andere Rechtsvorschriften über Geheimhaltung verletzt würden, oder

5. Vereinbarungen entgegenstehen, die mit Eigentümern aus Anlass der Übernahme getroffen wurden."

35 LT-Drs. 11/2802, S. 17 f.; in der Praxis hat $\$ 4$ Abs. 1 LArchG seit Inkrafttreten des LArchG 1990 keine große Rolle gespielt. Abgesehen von der Benutzung von Adoptionsunterlagen kam es nur ganz selten zu Anträgen auf Einsichtnahme von betroffenen Personen. Etwas häufiger gab es hingegen Anträge auf Benutzung von Kindern nach dem Tod ihrer Eltern gem. $\$ 4$ Abs. 3 LArchG Rh-Pf, die sich oft auf den Zeitraum 1933-1945 bezogen. 


\section{b) Art. 21 DSGVO}

Art. 21 Abs. 1 DSGVO eröffnet die Möglichkeit des Widerspruchs betroffener Personen gegen die Verarbeitung sie betreffender personenbezogener Daten.

Der Ausschluss der Verarbeitung personenbezogener Daten infolge eines Widerspruchs betroffener Personen würde eine Archivierung der entsprechenden Daten und damit die durch Art. 89 Abs. 3 DSGVO privilegierte Verwirklichung der spezifischen Zwecke unmöglich machen. Sie würde einen unvertretbaren Eingriff in die Archivierung als privilegierte Form der Verarbeitung bedeuten, der zu einer Verfälschung der Überlieferung führen würde. ${ }^{36}$ Beispielsweise würde damit eine Überlieferung von Gefangenenpersonalakten fraglich werden, da zumindest bei einer großen Zahl ehemaliger Gefangener von einem Löschungsbegehren ausgegangen werden darf. ${ }^{37}$ Dies würde Forschungen zum Strafvollzug anhand von Archivgut erheblich einschränken. Daher wurde in $\$ 4$ Abs. 1 S. 4 LArchG Rh-Pf ein Widerspruchsrecht nach Art. 21 Abs. 1 DSGVO ausgeschlossen.

\section{c) Art. 16 DSGVO}

Art. 16 DSGVO regelt das Recht auf Berichtigung fehlerhafter personenbezogener Daten. Eine Berichtigung fehlerhafter Daten in Archivgut kann zu einer unvollständigen und möglicherweise verfälschten Überlieferung führen. Wurden Verwaltungsentscheidungen aufgrund falscher personenbezogener Daten getroffen, ließe sich im Fall einer nachträglichen Berichtigung der falschen Daten nicht mehr erkennen, auf welcher Grundlage die jeweilige Entscheidung getroffen wurde. Der Datenbestand in einem Archiv darf allenfalls durch Hinweise modifiziert, aber nicht in seiner Substanz verändert werden, ${ }^{38}$ denn Archivgut dient in einem demokratischen Rechtsstaat u.a. zur retroperspektiven Kontrolle des Regierungs- und Verwaltungshandelns. Dadurch sind Archive auf ein hohes $\mathrm{Maß}$ an Authentizität und Vollständigkeit des archivierten Quellenmaterials angewiesen ${ }^{39}$, weswegen $\$ 7$ Abs. 1 LArchG Rh-Pf vorschreibt, dass öffentliche Stellen ihre Unterlagen den Archiven „unverändert“ zu übergeben haben. Eingriffe in die Substanz des Archivguts sind unzulässig. ${ }^{40}$

\4 Abs. 2 S. 3 LArchG Rh-Pf a.F. sah bereits eine Regelung zur Berichtigung vor, dass dem Archivgut eine Gegendarstellung beizufügen ist, wenn die betroffene Person

36 LT-Drs. 17/1067, S. 8.

37 Vgl. nur VG Darmstadt, Urt. v. 15.10.2003 - 5 E 1295/97 (3), NJW 2004, 1471.

38 LT-Drs. 17/1067, S. 8.

39 Birk, Sächsisches Archivblatt 2 2018, 11, 12. Die „Systemrelevanz der Archive“ wurde immer wieder von Heribert Prantl hervorgehoben: Hirnlosigkeit ist kein Geschäftsmodell, abrufbar unter https://www.sueddeutsche.de/politik/prantl-stasi-unterlagen-archive-1.460222 3, zuletzt abgerufen am 11.06.2018; vgl. ebenso: Kleifeld, Archive und Demokratie. Demokratische Defizite der öffentlichen Archive im politischen System der Bundesrepublik Deutschland, 2018, S. 95-115.

40 Hausmann, Landesarchivgesetz, S. 22.

RuZ 1. Jg. 2/2020 
die Unrichtigkeit personenbezogener Angaben glaubhaft machte. Ausgeschlossen ist dies bei rechtskräftigen gerichtlichen oder bestandskräftigen behördlichen Entscheidungen. ${ }^{41}$ Dadurch wurde bereits ein tragfähiger Kompromiss zwischen dem Interesse um die Darstellung ihrer Person in der „Nachwelt“ besorgten Betroffenen und den Interessen des Archivs geschaffen, das um die Integrität seiner Unterlagen bemüht ist. ${ }^{42}$ Diese bisherige Regelung greift nicht in dem Umfang in die Tätigkeit der Archive ein, wie es Art. 16 DSGVO tun würde; insbesondere haben Berichtigungen nicht unverzüglich zu erfolgen, sondern nur nach Glaubhaftmachung und Entscheidung der Stelle, bei welcher die Unterlagen entstanden sind. Zudem wird eine Berichtigung nur beigefügt und keine Veränderung am Original vorgenommen. ${ }^{43}$

\4 Abs. 2 S. 5 LArchG Rh-Pf schließt daher weitergehende Berichtigungsansprüche nach Art. 16 DSGVO aus.

\section{d) Art. 18 DSGVO}

Art. 18 EU-DSVO räumt betroffenen Personen ein Recht auf Einschränkung der Verarbeitung personenbezogener Daten ein.

Die Rechte der betroffenen Personen und der Schutz ihrer Interessen sind durch die geltenden archivgesetzlichen Bestimmungen bereits hinreichend gewahrt, insbesondere durch die für Archivgut allgemein und für personenbezogene Unterlagen im Besondere geltenden Sperrfristen, die sich in der Vergangenheit bereits bewährt haben, sowie durch die bereits erwähnten archivgesetzlichen Berichtigungsansprüche betroffener Personen, wenn diese die Richtigkeit von personenbezogenen Daten bestreiten. ${ }^{44} \mathrm{Zu}-$ dem besteht mit $\mathbb{3}$ Abs. 2 Nr. 2 LArchG Rh-Pf auf die bereits hingewiesene Auffangnorm, die auch schutzwürdige Belange betroffener Personen und Dritter berücksichtigt, die nicht durch die Sperrfristen nach $\$ 3$ Abs. 3 LArchG Rh-Pf geschützt sind. $\mathrm{Zu}$ denken ist hier z.B. an multipersonale Daten, wie sie bei erblichen Krankheiten vorliegen können. $\$ 4$ Abs. 2 S. 5 LArchG Rh-Pf schließt daher ebenfalls Ansprüche betroffener Personen nach Art. 18 DSGVO aus.

\section{e) Art. 19 DSGVO}

Art. 19 DSGVO sieht eine Mitteilungspflicht der Daten verarbeitenden Stelle gegenüber allen Empfängern, denen er personenbezogene Daten offenbart hat, bei Berichtigung oder Löschung personenbezogener Daten oder der Einschränkung der Verarbeitung vor, die auf Grundlage der Art. 16 bis Art. 18 DSGVO erfolgten. $\$ 4$ Abs. 2 S. 6

41 Entsprechende Anträge auf Berichtigung kommen in der Archivpraxis äußerst selten vor.

42 Manegold, Archivrecht. Die Archivierungspflicht öffentlicher Stellen und das Archivzugangsrecht des historischen Forschers im Lichte der Forschungsfreiheitsverbürgung des Art. 5 Abs. 3 GG, 2002, S. 321.

43 LT-Drs. 17/1067, S. 8.

44 LT-Drs. 17/1067, S. 9. 
LArchG Rh-Pf schließt daher nun Mitteilungspflichten nach Art. 19 DSGVO aus. Da Löschpflichten durch Art. 17 Abs. 3 lit.d DSGVO und das Recht auf Einschränkung durch $\ 5$ Abs. 2 S. 5 LArchG Rh-Pf bereits ausgeschlossen wurden, kämen vorliegend nur noch Mitteilungspflichten über die auf Grundlage von $\$ 4$ Abs. 2 S. 4 LArchG RhPf erfolgten Berichtigungen in Form von Beifügungen in Betracht. Da Berichtigungsansprüche, wie bereits erwähnt, äußerst selten vorkommen, bleibt fraglich, ob entsprechende Informationspflichten wirklich die Arbeitsfähigkeit der öffentlichen Archive ernsthaft gefährden, ${ }^{45}$ zumal keine Pflicht zur Mitteilung besteht, wenn und soweit diese unmöglich oder mit einem unverhältnismäßigem Aufwand verbunden sind. ${ }^{46} \mathrm{Im}$ Rückblick ist es daher zweifelhaft, ob eine Derogation von Art. 19 DSGVO überhaupt angezeigt war. ${ }^{47}$

\section{f) Art. 20 DSGVO}

Art. 20 DSGVO räumt betroffenen Personen ein Recht auf Datenübertragbarkeit ein. Auch wenn sich diese Vorschrift nur auf Daten bezieht, die die betroffene Person selbst einem Archiv zur Verfügung gestellt hat, was vor allem bei Vor- und Nachlässen der Fall sein dürfte, hätte eine Anwendung dieser Vorschrift auf Archive bzw. Archivgut zur Folge, dass Archive in größerem Umfang Datenverarbeitungsprozesse durchführen müssten: Alle von Art. 20 DSGVO erfassten Daten, die ausschließlich in analoger Form vorliegen, müssten in ein strukturiertes, gängiges und maschinenlesbares Format übertragen werden. Die Herstellung der Übertragbarkeit von Daten aus Archivgut ist jedoch nicht Aufgabe der Archive. Auch diese Verpflichtung würde somit dem Zweck der Archive widersprechen und angesichts der damit verbundenen Aufwände eine ernsthafte Beeinträchtigung der spezifischen Zwecke der Archivierung im Sinne des Art. 89 S. 3 DSGVO darstellen. Wie bereits erwähnt, wurden die Rechte betroffener Personen auf Auskunftserteilung oder Einsicht in sie betreffendes Archivgut im LArchG Rh-Pf bereits hinreichend geregelt. ${ }^{48}$

$\$ 4$ Abs. 2 S. 7 LArchG Rh-Pf sieht daher vor, dass eine Datenübertragbarkeit nach Art. 20 DSGVO nicht besteht.

\section{Schluss}

Mit der Änderung des Landesarchivgesetzes hat nun auch Rheinland-Pfalz für seine öffentlichen Archive eine datenschutz- und europarechtskonforme Rechtsgrundlage geschaffen, durch die einerseits gesichert ist, dass auch besondere Kategorien perso-

45 So LT-Drs. $17 / 1067$, S. 9.

46 Paal/Pauly-Paal, DSGVO/BDSG, 2. Auflage, 2017, Art. 19 Rn. 13.

47 Der Bund und Bayern haben in ihren Landesdatenschutzgesetzen auf eine entsprechende Derogation verzichtet, Sachsen und Niedersachsen haben eine solche hingegen aufgenommen.

48 LT-Drs. 17/1067, S. 7 f.

RuZ 1. Jg. 2/2020 
nenbezogener Daten verarbeitet werden dürfen und andererseits diverse Betroffenenrechte durch Derogation ausgeschlossen sind. Auch wurde abgewendet, dass Archive durch überhandnehmende und im Gegensatz zu archivischen Grundsätzen stehende datenschutzrechtliche Pflichten in ihrer Arbeit ernsthaft gefährdet werden. Inwiefern mit diesen Änderungen tatsächlich alle Vorgaben der DSGVO umgesetzt wurden, ist aktuell nur schwer einzuschätzen, da viele Regelungen, die auch Auswirkungen auf das Archivwesen haben, nicht eindeutig sind und noch der Klärung durch die Rechtsprechung und archiv- wie rechtspolitischer Diskussion bedürfen. ${ }^{49}$

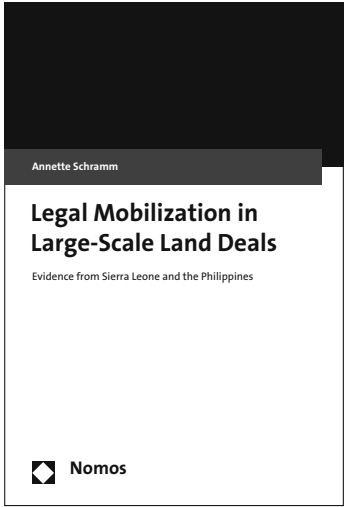

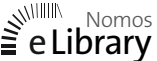

\section{Legal Mobilization in} Large-Scale Land Deals

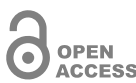

Evidence from Sierra Leone and the Philippines

Von Dr. Annette Schramm

2020, 294 S., brosch., 59,-€

ISBN 978-3-8487-6703-8

In englischer Sprache

Wie verteidigen lokale Gemeinschaften ihre Interessen gegen großflächige Landinvestitionen mit der Hilfe von rechtlichen Normen? Die Autorin beantwortet diese Frage durch vergleichende Fallstudien aus Sierra Leone und den Philippinen durch die Kombination verschiedener Forschungsperspektiven.

Bestellen Sie im Buchhandel oder versandkostenfrei online unter nomos-shop.de

49 Ähnliche Einschätzung bei: Heilmann, Die Anpassung des Niedersächsischen Archivgesetzes an die Vorgaben der Datenschutz-Grundverordnung, Archiv-Nachrichten Niedersachsen 23, 2018, 139, 144 und Birk, Sächsisches Archivblatt 2 2018, 11, 12. 